\title{
FRET from CdSe/ZnS Core-Shell Quantum Dots to Fluorescein 27 Dye
}

\author{
Math A. Shivkumar ${ }^{1}$, Laxmi S. Inamdar (Doddamani) ${ }^{2}$, Mohammad Hussain K. Rabinal ${ }^{1}$, \\ Basavaraj G. Mulimani ${ }^{1}$, Gopal M. Advi Rao ${ }^{3}$, Sanjeev R. Inamdar ${ }^{1^{*}}$ \\ ${ }^{1}$ Laser Spectroscopy Programme, Department of Physics, Karnatak University, Dharwad, India \\ ${ }^{2}$ Molecular Endocrinology and Development Laboratory, Department of Zoology, Karnatak University, Dharwad, India \\ ${ }^{3}$ Department of Biochemistry, Davangere University, Davangere, India \\ Email: "him_lax3@yahoo.co.in
}

Received November 18, 2012; revised December 20, 2012; accepted January 21, 2013

\begin{abstract}
Semiconductor QDs have emerged as a novel class of fluorophore with unique photoluminescence properties, in particular, $\mathrm{CdSe} / \mathrm{ZnS}$ core-shell QDs have been successfully used as biocompatible fluorescence resonance energy transfer donors. Here we report FRET between CdSe/ZnS core-shell QDs (donor) and organic dye fluorescein 27 (F27) (acceptor). The results demonstrate the occurrence of efficient energy transfer in the system and the FRET efficiency is not only influenced by the spectral overlap between the QD donor emission and acceptor absorption, it might depend on QDs surface effect also. Efforts are made to correlate quantitatively spectral dependence of FRET rate with acceptor absorption spectrum, Forster distance, transfer efficiency (E) obtained employing steady-state \& time-resolved technique.
\end{abstract}

Keywords: Quantum Dot; FRET; Transfer Efficiency; Spectral Overlap; Forster Distance

\section{Introduction}

Contemporary research interest in semiconductor nano particles or quantum dots (QDs), the tiny light emitting particles on the nanometer scale, focuses on their application as biological reporters not only because of their size tunable properties but also due to the fact that they have virtually the same dimension as biological macromolecules such as nucleic acids and proteins [1,2]. This similarity in dimension helps in integration of nanoparticles with biomolecules leading to varied applications in medical diagnostics, targeted drug delivery, etc. [3]. Such applications necessitate energy or charge-carrier transfer between quantum dots and the molecular species [4]. Quantum dots have many unique properties and exhibit interesting phenomena such as size dependent emission wavelength, narrow emission peak, broad excitation range, high photochemical stability and improved photoluminescence quantum yield upon passivating the surface traps with higher band gap semi conductors such as $\mathrm{ZnS}$ [5-10]. When these particles are photoexcited, electron-hole pairs are generated and upon their recombination fluorescence light is emitted due to the small size quantum effect playing an important role. This behaviour is quite useful in fluorescence resonance energy

"Corresponding author. transfer (FRET) between groups of molecules or complexes. The study of successful use of QDs as donors in FRET has been reported in developing chemical and biosensors [11-16]. FRET is the distance dependent transfer of non radiative electronic excitation energy from a donor molecule to an acceptor molecule and due to its sensitivity to distance it has been used to investigate molecular interactions. FRET process generally proceeds through dipole-dipole coupling between the transition moments of the two fluorophores viz., the donor and the acceptor (both fluorescent dyes as well as QDs). Thus it is proportional to the square of the coupling and hence varies rapidly with the distance $(\mathrm{R})$ between the donor and acceptor as inverse sixth power of $\mathrm{R}$. This strong dependence on distance makes FRET a very useful tool to investigate distances in the range of 20 $100 \AA[4,17-22]$. The transfer of energy manifests in a reduction in the donor fluorescence intensity, the excited state lifetime and a simultaneous increase in the acceptor emission intensity. A pair of molecules, wherein FRET occurs in such a manner, is often referred to as donoracceptor pair; and the efficiency of the FRET process depends on the separation between the donor and acceptor [23,24]. There are quite a few reports of use of FRET in semiconductor QDs and biomolecules such as dye labelled proteins in order to gain information on 
conjugate structure and valence the number of biomolecules attached and to monitor them inside the cells [25].

FRET between QDs and a fluorescent protein has been reported in literature [19-24] that demonstrate the dependence of energy transfer efficiency on the spectral overlap and the conjugate valence. While good spectral overlap is of paramount importance to high transfer efficiency, the donor and acceptor photoluminescence signals must also be well resolved to extract accurate experimental information for the system under investigation $[15,26]$. It has also been possible to assemble hybrid nanoparticle-protein/DNA comprising QDs simultaneously coupled to dye labelled proteins, oligonuceotides and dyes [27,28]. This study also reported the occurrence of FRET beyond $100 \AA$. Medintz et al. [29,30] have demonstrated FRET using CdSe-ZnS (core-shell) QD donors self assembled with three distinct fluorescent protein acceptors. Water soluble CdSe-ZnS QDs covalently attached to human immunoglobulin $\mathrm{G}(\mathrm{IgG})$ have been shown to act as efficient FRET donors for fluorescent isothiocyanate (labelled with goat antihuman $\mathrm{IgG}$ ) [31-33]. The CdSe-ZnS QDs have also been used for the development of self-assembled donor for long range FRET in a 3-chromophore FRET system. Muthugan et al. [34] have reported efficient FRET between CdSe-ZnS nanocrystals and Rhodamine $\mathrm{B}$ for light harvesting in solution by utilizing the electrostatic interaction between them. These authors showed that the $\mathrm{ZnS}$ capped CdSe core-shell nanocrystals are sensitive to external factors such as $\mathrm{pH}$ and divalent cations, leading to potential applications in optical sensing and homogeneous assays. Thus, with a view to initiate research on development of quantum dot based FRET chemical and biosensors, we have taken up FRET study between two sizes of CdSe$\mathrm{ZnS}$ core-shell QDs as donors and an organic dye (fluorescein 27, F27) as acceptor employing various spectral methods viz., uv/vis absorption spectroscopy, steady state and time resolved fluorescence spectroscopy. The paper aims at understanding - how the energy transfer efficiency and Forster distance depend on the varying size of the QD donor.

\section{Theory}

Forster developed the quantitative theory for resonance energy transfer in the late 1940s. Hence, the process is often called Forster resonance energy transfer [20,35]. FRET is a photo physical process where the excited-state energy from a donor molecule is transferred non-radiatively to an acceptor molecule (in the ground state) at a close distance via weak dipole-dipole coupling [16].

Forster derived the following expression for the rate constant of transfer, $k_{\mathrm{T}}$ :

$$
k_{\mathrm{T}}(R)=\frac{1}{\tau_{D}}\left(\frac{R_{0}}{r}\right)^{6}
$$

where $r$ is the distance between donor and acceptor molecules and $\tau_{D}$ is the fluorescence lifetime of the donor (without acceptor). Since the transfer rate is proportional to the inverse 6 th power of the distance $(R)$, it is an extremely sensitive parameter for obtaining distances between 1 and $10 \mathrm{~nm}$. The distance at which the excitation energy of the donor is transferred to the acceptor with a probability of 0.5 is called Forster or critical distance $\left(R_{0}\right)$ and can be calculated using the relevant spectroscopic properties of the participating molecules:

$$
R_{0}^{6}=\frac{9000 \operatorname{In}(10) k^{2} \varphi_{0}}{128 \pi^{5} N_{A} n^{4}} \int_{0}^{\infty} \frac{F_{D}(\lambda) \varepsilon_{A}(\lambda)}{\lambda^{4}} \mathrm{~d} \lambda
$$

where $k^{2}$ is the molecular orientation factor, $\varphi_{D}$ is the quantum yield of donor fluorescence (in the absence of acceptor), $N_{A}$ is Avogadro's number and $n$ is the refractive index of the intervening medium [36]. The integral in the above equation $\left(J, \mathrm{M}^{-1} \cdot \mathrm{cm}^{3}\right)$ provides the degree of spectral overlap between donor fluorescence spectrum $F_{D}(\lambda)$ and acceptor absorption spectrum $\varepsilon_{A}(\lambda)$, given in either wave number $(v)$ or wavelength $(\lambda)$ scale:

$$
J(\lambda)=\int_{0}^{\infty} F_{D}(\lambda) \varepsilon_{A}(\lambda) \lambda^{-4} \mathrm{~d} \lambda
$$

The orientation factor $k^{2}$ is given by [37]

$$
\begin{aligned}
k^{2} & =\left[\cos \theta_{T}-3 \cos \theta_{D} \cos \theta_{A}\right]^{2} \\
& =\left[\sin \theta_{D} \sin \theta_{A} \cos \varphi-2 \cos \theta_{D} \cos \theta_{A}\right]^{2}
\end{aligned}
$$

where $\theta_{T}$ is the angle between the transition moments of donor and acceptor, $\theta_{D}$ and $\theta_{A}$ are the angles of the transition moments of donor and acceptor with the separation vector and $\varphi$ is the angle between the two planes formed by the transition moments of donor and acceptor and the separation vector. For systems without any three-dimensional, spatial information the orientation factor is an indeterminate parameter in the evaluation of $R_{0}$, having any value $0<k^{2}<4$. All other parameters can be measured or evaluated. Forster also introduced the transfer efficiency $E$, which is only a function of actual $(r)$ and critical $\left(R_{0}\right)$ distances:

$$
E=\frac{1}{1+\left(\frac{r}{R_{0}}\right)^{6}}
$$

Among the several methods available for quantification of FRET, the one based on donor fluorescence lifetimes is the most straightforward, as the fluorescence lifetime is a concentration-independent property, while 
the fluorescence intensity is not. Fluorescence lifetimes of the donor in the absence $\left(\tau_{D}\right)$ and presence $\left(\tau_{D A}\right)$ of acceptor molecules are often measured for the observation of FRET and a decreased fluorescence lifetime of the donor is then an indication of molecular interactions. From this reduction in lifetimes one obtains the experimental FRET efficiency $(E)$ :

$$
E=1-\frac{\tau_{D A}}{\tau_{D}}
$$

If an acceptor molecule is brought close to a donor molecule within a range of $20-100 \AA$, the fluorescence intensity of the acceptor molecule increases, while that of the donor molecule decreases, due to an increase in the efficiency of FRET from donor to acceptor molecule $[13,20,22,26] . E$ can be estimated by both steady-state and time resolved measurements, respectively, using Equations (7) and (8),

$$
E=1-\frac{F_{D A}}{F_{D}}
$$

where $F_{D A}$ is fluorescence intensity of the donor with acceptor, and $F_{D}$ is that without acceptor, and

$$
E=1-\frac{\tau_{D A}}{\tau_{D}}
$$

The transfer efficiency and the distance between the donor and acceptor, $R$, are related by the equation,

$$
r=R_{0}(1 / E-1)^{1 / 6}
$$

The magnitude of $R_{0}$ depends on the spectral properties of the donor and acceptor

$$
R_{0}=\left[8.8 \times 10^{23} K^{2} n^{-4} \varphi_{D} J\right]^{1 / 6}
$$

where $\varphi_{D}$ is quantum yield of the donor in the absence of acceptor.

\section{Materials and Methods}

Fluorescence spectra of the donor (QDs), acceptor (dye) and the mixed binary solutions were recorded using Hitachi F7000 spectrofluorometer and the uv/vis absorption spectra were obtained using Hitachi U2800 spectrophotometer. The fluorescence lifetimes were measured employing picosecond time domain spectrometer based on Time Correlated Single Photon Counting (TCSPC) technique (IBH Jobin Yvon6.1), described elsewhere [38]. The samples of CdSe/ZnS $480 \mathrm{~nm}$ (2.5 nm size, QD1) and $510 \mathrm{~nm}(3.0 \mathrm{~nm}$ size, QD2) quantum dots were excited at $375 \mathrm{~nm}$ using Nano LED in an IBH Fluorocube apparatus. The fluorescence emission at the magic angle $54.7^{\circ}$ was dispersed in a monochromator $(\mathrm{f} / 3)$ aperture and counted by a Hamamatsu Micro Channel Plate Photo-multiplier tube (R3809 MCP-PMT). The instru- ment response function for this system is $\sim 52 \mathrm{ps}$. An iterative fitting program provided by IBH (DAS-6) analyzed the fluorescence decay curves.

Toluene solutions of $\mathrm{CdSe} / \mathrm{ZnS}$ core-shell (Lumidot) QDs [480 nm $(2.5 \mathrm{~nm}$ size $)$ and $510 \mathrm{~nm}(3.0 \mathrm{~nm}$ size $)]$ were purchased from Sigma-Aldrich Chemicals Co. and Fluorescein 27 dye from Lambdaphysik, Inc. All the solvents used were of HPLC grade and were used without further purification. The quantum yields $(\varphi)$ of the QDs were obtained by comparison with Coumarin 307 dye as a standard reference [39] in ethanol using the equation [40],

$$
\varphi=\varphi_{R} \frac{\mathrm{I}}{\mathrm{I}_{R}} \frac{\mathrm{OD}_{R}}{\mathrm{OD}} \frac{n^{2}}{n_{R}^{2}}
$$

where I is the integrated intensity, OD is the optical density and $\mathrm{n}$ is the refractive index, the subscript $R$ refers to the reference fluorophore of known quantum yield. Time resolved fluorescence measurements provide significant and precise information about the molecular interactions than the steady state measurements. By monitoring the fluorescence lifetime of quantum dots in the presence and absence of the dye, important information can be obtained on molecular interactions. The effect of dynamic quenching and in particular the processes of FRET are now widely studied in photochemistry and photobiology, wherein, signal intensity is often used to quantify FRET efficiency, but fluorescence lifetime is more precise and robust technique because it is independent of concentration, and is thus a desirable parameter for these measurements.

\section{Results and Discussion}

\subsection{Steady-State Measurements}

With a view to develop FRET chemical and biosensors based on semiconductor QDs, two sets of QDs of sizes 480 (QD1) and $510 \mathrm{~nm}$ (QD2) are chosen as donors in the present study with Fluorescein 27 dye as acceptor molecule. Each molar concentration was studied in free solution under steady state condition. Figure 1(A) shows the normalized emission spectra of $\mathrm{CdSe} / \mathrm{ZnS}$ core-shell quantum dots in toluene excited with $370 \mathrm{~nm}$ light along
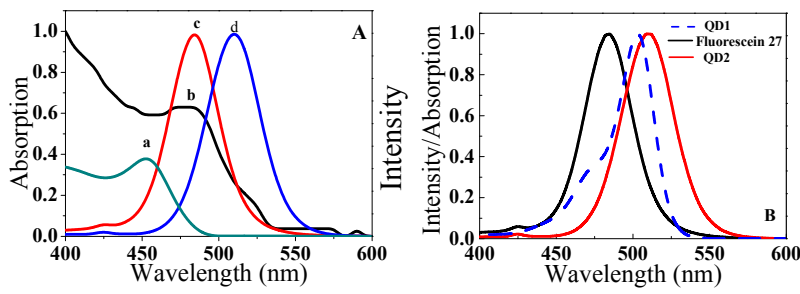

Figure 1. (A) Absorption and emission spectra of QD1 (a, c) and QD2 (b, d); (B) overlap of Emission spectra of QD1 and QD2 with absorption spectrum of F27 dye. 
with the absorption spectrum of F27 dye. F27 dye does not exhibit any appreciable absorption at $370 \mathrm{~nm}$ and therefore allows the exclusive excitation of the donor. The photon induced energy transfer from donor to acceptor is monitored. A necessary factor to achieve FRET phenomena is good spectral overlap between the absorption of acceptor and emission spectrum of donor. Figure 1(B) shows the spectral overlap function for both the sets of CdSe/ZnS QDs and F27 dye. It may be noted that the spectral overlap is better for QD2 + F27 pair. It is observed that the addition of F27 dye to the $\mathrm{CdSe} / \mathrm{ZnS}$ solution does not lead to any change in the absorption spectrum of QDs.

Figures 2(a) and (b) show fluorescence spectra of $\mathrm{CdSe} / \mathrm{ZnS}$ in the presence of varying concentration of F27 dye. Upon addition of F27 dye a gradual and significant quenching of the donor (CdSe/ZnS QDs) fluorescence was observed with a simultaneous enhancement in the acceptor emission indicating some form of energy transfer to acceptor (F27). Consequently, with the addition of the acceptor in increased proportion results in the presence of more acceptor molecules. From Figures 2(a) and (b) it may be noted that a strong size dependent quenching is occurring. Hence, this quenching of QD emission and simultaneous enhancement in F27 emission validates and confirms the nonradiative resonance energy transfer from CdSe/ZnS QD to F27 dye. The generously proportioned quenching of donor and the use of high acceptor to QD ratios will compensate the large separation distance which improves the FRET efficiency.

The rate of quenching of donor emission is illustrated by the decrease in average lifetime. It reveals that transfer efficiency will depend on the size of the particle and spectral overlap of donor and acceptor. Quenching of donoremission as function of varying concentration of acceptor is expected and is described by the well known Stern-Volmer Equation

$$
\frac{F_{0}}{F}=1+k_{q} \tau_{0}[Q]=1+K_{S V}[Q]=\frac{\tau_{o}}{\tau}
$$

where $F_{0}$ and $\mathrm{F}$ are the fluorescence intensities in the absence and presence of quencher, respectively, $[Q]$ is
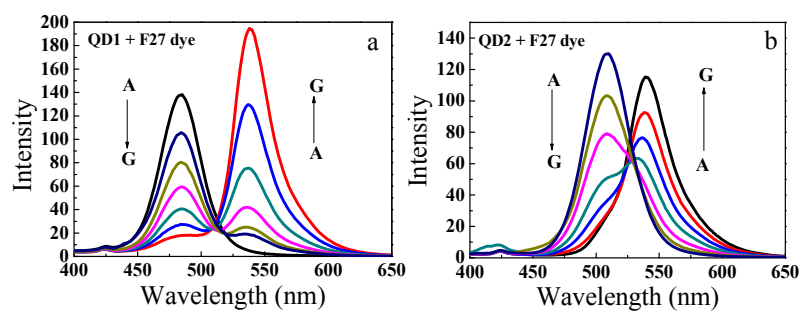

Figure 2. Fluorescence spectra of (a) QD1 and (b) QD2 (1.0 $\times 10^{-3} \mathrm{M}$ ) in the presence of varying concentrations of F27 dye (acceptor); A) 0 ; B) $0.8 \times 10^{-6} \mathrm{M}$; C) $1.0 \times 10^{-6} \mathrm{M}$; D) $3.0 \times 10^{-6} \mathrm{M}$; E) $6.0 \times 10^{-6} \mathrm{M}$; F) $8.0 \times 10^{-6}$, G) $10 \times 10^{-6} \mathrm{M}$. the concentration of quencher, $\tau_{0}$ and $\tau$ are the lifetimes in the absence and presence of quencher, respectively. Since this quenching is an additional rate process which depopulates the excited state, the above equation illustrates the important characteristics of quenching, which is equivalent to decrease in fluorescence intensity and in life time. A linear Stern-Volmer plot of $\mathrm{F}_{0} / \mathrm{F}$ versus quencher concentration is shown in Figures 3(A) and (B) and from the slope of plot, $K_{\mathrm{SV}}$ was found to be 0.534 $\times 10^{6}$ and $0.474 \times 10^{6} \mathrm{M}^{-1}$ for the QD1-F27 and QD2-F27 systems, respectively. The estimated values of quenching rate parameters $\left(k_{\mathrm{q}}\right)$ are $4.07 \times 10^{13}$ and $4.09 \times$ $10^{13} \mathrm{M}^{-1} \cdot \mathrm{s}^{-1}$ for the QD1-F27 and QD2-F27, respectively. A decrease in fluorescence lifetimes of the QDs is equivalent to $\mathrm{F}_{0} / \mathrm{F}$. The FRET parameters in steady state measurement were estimated by using Equations (1)-(12) (Table 1).

\subsection{Time-Resolved Measurements}

The nonradiative resonance energy transfer is known to substantially alter the exciton lifetime properties of the donor $\left(\tau_{D}\right)$. Thus, the energy transfer process was further examined using fluorescence decay analysis by TCSPC Technique. Figure 4 shows the fluorescence decay curves of QDs with and without F27 dye. The lifetimes in the presence and absence of acceptor dye are likely to throw light on the FRET efficiency in the chosen donor-acceptor pairs. The samples were excited with 375 nm light from LEDs and fluorescence decay traces required a sum of three exponential functions to reach low $\chi^{2}$ values as well as random distributions of the weighted residuals, which are indicators of the goodness of the fits.

$$
I(t)=A_{1} \mathrm{e}^{-t / \tau_{1}}+A_{2} \mathrm{e}^{-t / \tau_{2}}+A_{3} \mathrm{e}^{-t / \tau_{3}}
$$

where $\tau_{1}, \tau_{2}$ and $\tau_{3}$ are the time constants and $\mathrm{A}_{1}, \mathrm{~A}_{2}$, and $\mathrm{A}_{3}$ are the normalized amplitudes of the components, respectively. An average amplitude-weighted lifetime is defined as [31]

$$
\tau=\left(A_{1} \tau_{1}+A_{2} \tau_{2}+A_{3} \tau_{3}\right) .
$$

Decay curves of the two CdSe/ZnS core-shell QD are
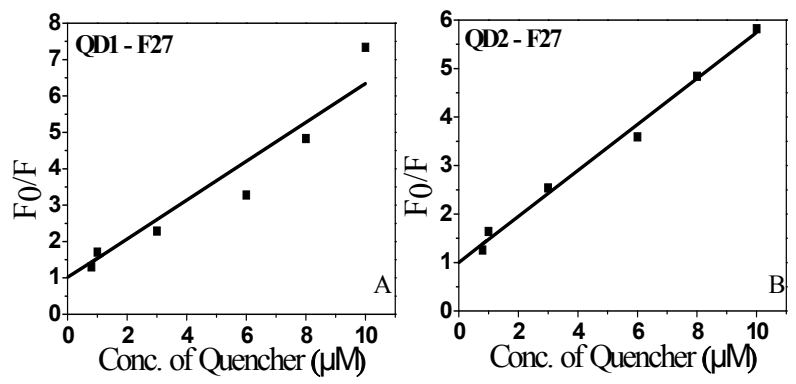

Figure 3. Steady state Stern-Volmer plots of Fo/F vs. concentration of quencher: (A) QD1 and (B) QD2. 
Table 1. FRET parameters obtained from steady state measurements.

\begin{tabular}{|c|c|c|c|c|c|c|c|}
\hline System & $\mathbf{J}(\lambda)\left(\mathbf{M}^{-1} \cdot \mathrm{cm}^{-1} \cdot \mathrm{nm}^{4}\right)$ & E (\%) & $\mathbf{E}^{*}(\%)$ & $\mathbf{R}_{0}(\AA)$ & $\mathbf{r}(\AA)$ & $k_{\mathrm{T}}(\mathbf{r}) \mathrm{s}^{-1}$ & Decay Rate $\Gamma \mathrm{s}^{-1}$ \\
\hline QD1 + F27 & $3.216 \times 10^{-14}$ & 52.77 & 51.26 & 34.62 & 19.16 & $4.24 \times 10^{9}$ & $7.62 \times 10^{7}$ \\
\hline QD2 + F27 & $4.619 \times 10^{-14}$ & 52.93 & 43.78 & 35.84 & 20.02 & $3.75 \times 10^{9}$ & $8.63 \times 10^{7}$ \\
\hline
\end{tabular}

*Efficiency calculated from time resolved data.
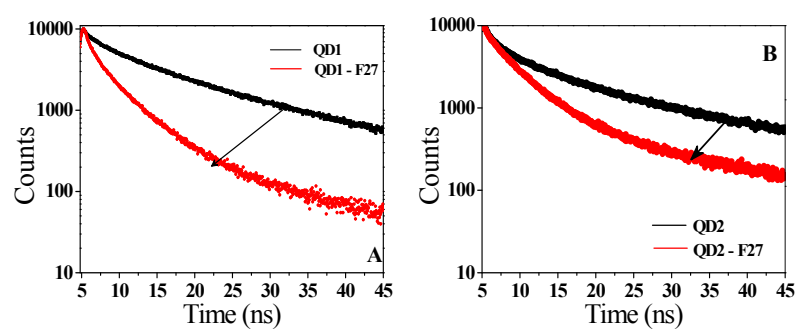

Figure 4. Log scale plot of time-resolved PL trace for (A) QD1 and (B) QD2 with and without F27 dye (donor emission wavelength fixed at 480 and $510 \mathrm{~nm}$ with excitation at 375 nm).

multiexponential with three decay components shown in Table 2. The photoluminescence decay of QD1 is triexponential with decay components of 506.3 ps (0.02) $\left(\tau_{1}\right), 4.26 \mathrm{~ns}(0.14)\left(\tau_{2}\right)$ and $15.04 \mathrm{~ns}(0.84)\left(\tau_{3}\right)$ with the average decay time being $13.12 \mathrm{~ns}$, according to Equation (14). However, its average decay time in the presence of the acceptor is $6.49 \mathrm{~ns}$. The values of decay times for the QD2 are 483.4 ps $(0.05)\left(\tau_{1}\right), 3.36 \mathrm{~ns}(0.17)$ $\left(\tau_{2}\right)$ and $14.10 \mathrm{~ns}(0.78)\left(\tau_{3}\right)$ with the average decay time being $11.58 \mathrm{~ns}$. In the presence of the dye F27, however, it decreases to $6.51 \mathrm{~ns}$. Thus the lifetimes of the both the QDs are significantly shortened upon addition of the F27 dye and it is one of the characteristic features of efficient energy transfer in the donor-acceptor systems. The reduction in lifetime also heralds the quenching of the QD emission by the acceptor dye molecules. SternVolmer plots of $\tau_{0} / \tau$ versus quencher concentration shown in Figure 5 exhibit linear trends and from the slopes of these plots, $K_{\mathrm{SV}}$ was found to be $0.099 \times 10^{6}$ $\mathrm{M}^{-1}$ and $0.3372 \times 10^{6} \mathrm{M}^{-1}$ for the QD1-F27 and QD2-F27, respectively. The estimated quenching rate constants $\left(k_{\mathrm{q}}\right)$ are $7.545 \times 10^{12}$ and $2.911 \times 10^{13} \mathrm{M}^{-1} \cdot \mathrm{s}^{-1}$ for QD1-F27 and QD2-F27 systems, respectively. The expected value for the diffusion controlled bimolecular processes is $\sim 10^{10} \mathrm{M}^{-1} \cdot \mathrm{s}^{-1}$. As the experimentally obtained quenching rate constants are higher than the expected quenching constant, it is inferred that this is not a diffusion-controlled process.

Note that, the origin of multi-exponential emission decay of semiconductor quantum dots still remains a matter of debate. The fastest decay components reflect the emission from radiative relaxation of the excited electrons to the ground state and can be attributed to the initially populated core-state recombination [41]. A dis-
Table 2. FRET parameters from time-resolved measurements.

\begin{tabular}{ccccc}
\hline System & $\boldsymbol{\tau}_{\mathbf{1}}(\mathbf{p s})\left(\mathbf{a}_{1}\right)$ & $\boldsymbol{\tau}_{\mathbf{2}}(\mathbf{n s})\left(\mathbf{a}_{2}\right)$ & $\boldsymbol{\tau}_{3}(\mathbf{n s})\left(\mathbf{a}_{3}\right)$ & $<\boldsymbol{\tau}>\mathbf{n s}$ \\
\hline QD1 & $506.3(0.02)$ & $4.26(0.14)$ & $15.04(0.84)$ & 13.12 \\
QD1 + F27 & $559.5(0.13)$ & $3.21(0.42)$ & $11.18(0.45)$ & 6.49 \\
QD2 & $483.4(0.06)$ & $3.36(0.16)$ & $14.10(0.78)$ & 11.58 \\
QD2 + F27 & $301.8(0.07)$ & $3.65(0.57)$ & $12.16(0.37)$ & 6.51 \\
\hline
\end{tabular}

$\mathrm{a}_{1}, \mathrm{a}_{2}, \mathrm{a}_{3}$ are the normalized amplitudes components.
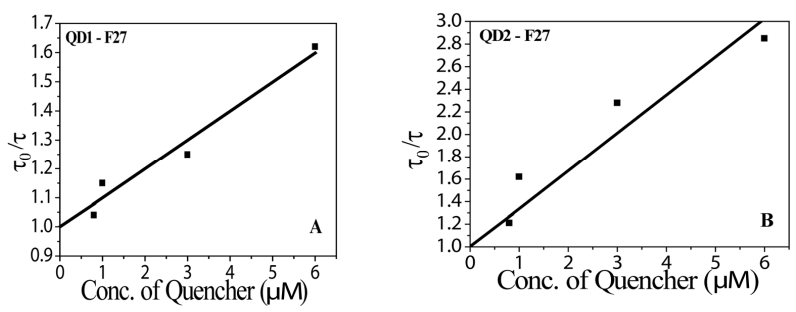

Figure 5. Time-resolved Stern-Volmer plots of $\tau_{0} / \tau$ vs. concentration of quencher: (A) QD1 and (B) QD2.

tribution in decay times is expected as a result of the variation in the nonradiative decay rates for different QDs due to variation in type and number of the quenching centres. A combination of all these processes, along with difference between the individual nanocrystals in population, gives rise to multi-exponential emission dynamics that result in a spread of luminescence lifetimes for different quantum dots $[42,43]$. Further, from the decay component data (Table 2) the observed results shows that the larger the value of $a_{3}$, the better the surface condition (with fewer defect states) and the larger role of surface related emission, since smaller QDs are known to have more surface trap states compared to the larger dots (higher surface to volume ratio and surface tension). It can be noted that, the shorter life time components in decay profile is due to the intrinsic recombination of initially populated core states and the longer lifetime component is attributed to the involvement of surface states in the carrier recombination process $[44,45]$. The longest and fastest decay times observed are $11.18 \mathrm{~ns}$ and 559.5 ps respectively, for QD1-F27 system, and $12.16 \mathrm{~ns}$ and $301.8 \mathrm{ps}$, respectively, for QD2-F27 systems.

We also examine the dependence of FRET efficiency 
upon the QD emission overlaps and whether the QD emission overlaps with red or blue regions of the acceptor absorption spectrum (Figure 4). The estimated results of steady-state measurements exhibit increased spectral overlap and efficiency in case of QD2-F27 as compared to the QD1-F27 pair, and also the energy transfer efficiency. When comparing this kind of transfer efficiency with time-resolved estimation, QD2-F27 pair exhibited smaller energy transfer efficiency as opposed to QD1F27. It's possible to describe this evidently by noticing spectral overlap of donor QDs and acceptor, i.e., QD1F27 pair exhibits some sort of redder counterpart of QD emission overlapping with bluer counterpart of F27 acceptor, leading to redder QDs population being quenched to greater extent which could result in a noticeable blueshift in the QDs emission. The obtained intermolecular distance and Forster Distance $\left(R_{0}\right)$ from these measurements are $19.16 \AA$ and $34.62 \AA$, respectively.

Similarly for QD2-F27 pair, bluer parts of QD2 emission overlap with the redder counterpart of acceptor $a b-$ sorption. Thus the redder counterparts of QDs emission participate in lesser efficient interactions compared to the blue counterpart. As a result difference in spectral overlap causes a noticeable redshift within the QDs ensemble emission. Values of $20.01 \AA$, and $35.84 \AA$ for intermolecular distance $(\mathrm{r})$ and Forster distance $\left(R_{0}\right)$, respectively, were obtained from this measurement which is consistent with the previous estimation.

Hence it reveals that the effect of spectral overlap between QDs emission and dye absorption is dominant. However, the pairs presented here show that the spectrally dependent dynamics changes are due to FRET and it is important to note that a decrease in efficiency even with a better spectral overlap is due to the heterogeneity in the $\lambda$-dependent FRET rate and acceptor absorption spectra [46].

Similar to these steady state measurements to analyze the effect of size and wavelength reliant variation upon FRET, we have measured the donor exciton lifetime over the emission spectrum (for different emission wavelength) of QDs population for each FRET pair without and with acceptor.

Figure 6 shows the time variation with the QD1-F27 pair which evidently reveals how the FRET dynamics are certainly not homogeneous within the QD population, where the QD emission overlaps with blue edge of the acceptor absorption, the decay rate becomes faster with increasing emission wavelength. Consequently the redder QDs possess better overlap with the acceptor absorption than the bluer QDs, which in turn results in a gradually faster FRET dynamics as we move through the bluer QDs to redder QDs.

In the second pair QD2-F27 the exciton decay becomes gradually slower as we move from the bluer QDs to the redder QDs, because in this pair the bluer counter-
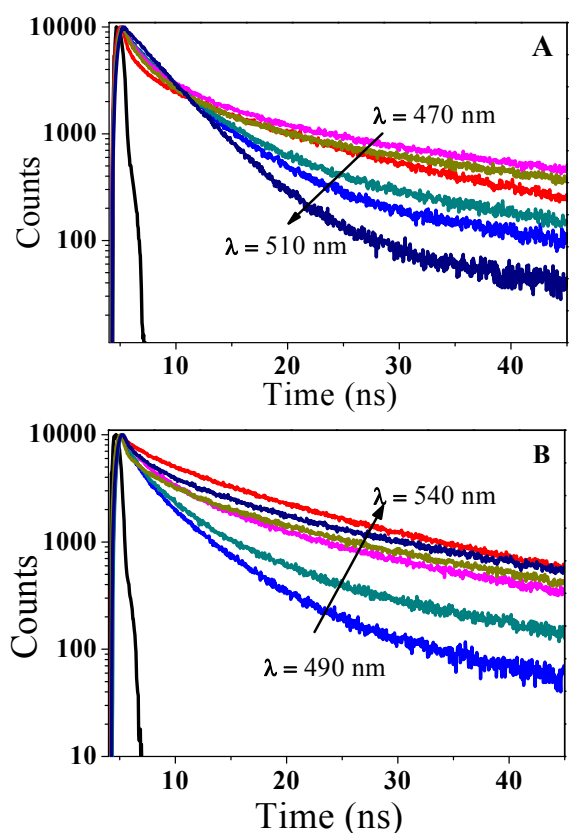

Figure 6. Plot of time-resolved decays of QD-F27 for different emission wavelengths selected: (A) QD1-F27 FRET pair, from $470 \mathrm{~nm}$ to $510 \mathrm{~nm}$; (B) QD2-F27 FRET pair, from $490 \mathrm{~nm}$ to $540 \mathrm{~nm}$.

parts possess a better spectral overlap than the redder QDs.

The spectral dependence of the FRET rate extracted from the time-resolved data is more precise since it is concentration independent and it is clear that heterogeneity in FRET rates are due to difference in spectral overlap within the same QD population. This is reflected in the values of $R_{0}$ and $E$ for the two FRET pairs (Table 2).

\section{Conclusions}

The higher potential of the QDs-dye nano assembly combination than the typical organic donor and acceptor dye pairs enhance the scope of FRET analysis particularly in biological applications. In this study we have explored the energy transfer between two sizes of $\mathrm{CdSe} / \mathrm{ZnS}$ QDs $(2.5 \mathrm{~nm}$ and $3.0 \mathrm{~nm}$ ) as donor and F27 dye as a common acceptor. The results clearly illustrate the dependence of FRET efficiency on spectral overlap as depicted in Figure 4. The two different sized QDs display different emission spectra and as the emission of QDs shifts to longer wavelengths the overlap with the absorption spectra of F27 dye increases. Consequent to increase in the QD size, the QDs undergo very different FRET quenching efficiency due to difference in their spectral overlap. The energy transfer rate between optimally resonant donor-acceptor pair estimated from steady state and time resolved study elucidate that the transfer rates estimated (at $4.24 \times 10^{9} \mathrm{~s}^{-1}$ and $3.75 \times 10^{9}$ 
$\mathrm{s}^{-1}$, respectively, for QD1 and QD2 CdSe/ZnS nanostructures) are much faster than the respective decay rates of $7.62 \times 10^{7} \mathrm{~s}^{-1}$ for QD1 and $8.63 \times 10^{7} \mathrm{~s}^{-1}$ for QD2. Hence, it is clearly seen that energy transfer is highly efficient $(43 \%-51 \%)$ over a range of $34-36 \AA\left(\mathrm{R}_{0}\right)$. The present study also demonstrates that such QD-dye pairs can act as very sensitive chemical sensors.

Further, we are interested in studying the importance of QDs in FRET by examining their optical properties in a quest to explore their possible subsequent applications in life sciences. Several technical challenges are posed towards their use in aqueous and hence biological environment. Here FRET is dependent on the type and size of QDs, several physical and chemical variables and surrounding environment viz., surface treatment and insolubility in water. This does become important as recently Parchur et al. [47] have shown that the luminescence intensity improves significantly after core-shell formation due to extent of decrease of nonradiative rates arising from surface dangling bonds and capping agent during the study of $\mathrm{Tb}^{3+}$-doped $\mathrm{CaMoO}_{4}$ core and core-shell nanoparticles.

\section{Acknowledgements}

The authors gratefully acknowledge the partial financial support from the University Grants Commission, New Delhi (under CPEPA, CAS, MRP) and DAE-BRNS (under MRP), Mumbai. MAS thanks the UGC for fellowship under RFSMS. Authors also thank Prof. P. Ramamurthy, National Centre for Ultrafast Processes, Chennai for time resolved measurements.

\section{REFERENCES}

[1] S. A. E. Marras, F. R. Kramer and S. Tyagi, "Efficiencies of Fluorescence Resonance Energy Transfer and Contact-Mediated Quenching in Oligonucleotide Probes," Oxford University Press, Nucleic Acids Research, Vol. 30, No. 21, 2002, p. e122. doi:10.1093/nar/gnf121

[2] J. A. Kloepfer, S. E. Bradforth and J. L. Nadeau, "Photophysical Properties of Biological Compatibility CdSe Quantum Dot Structures," Journal of Physical Chemistry $B$, Vol. 109, No. 20, 2005, pp. 9996-10003. doi:10.1021/jp044581g

[3] X. H. Gao, C. W. Warren Chan and S. Nie, "QuantumDot Nanocrystals for Ultrasensitive Biological Labeling and Multicolour Optical Encoding," Journal of Biomedical Optics, Vol. 7, No. 4, 2002, pp. 532-537. doi: $10.1117 / 1.1506706$

[4] K. E. Sapsford, L. Berti and I. L. Medintz, "Materials for Fluorescence Resonance Energy Transfer Analysis: Beyond Traditional Donor-Acceptor Combinations," Angewandte Chemie International Edition, Vol. 45, No. 28, 2006, pp. 4562-4588. doi:10.1002/anie.200503873

[5] A. Henglein, "Small-Particle Research: Physicochemical
Properties of Extremely Small Colloidal Metal and Semiconductor Particles," Chemical Reviews, Vol. 89, No. 8, 1989, pp. 1861-1873. doi:10.1021/cr00098a010

[6] A. P. Alivisatos, "Semiconductor Clusters, Nanocrystals, and Quantum Dots," Science, Vol. 271, No. 5251, 1996, pp. 933-937. doi:10.1126/science.271.5251.933

[7] B. O. Dabbousi, J. Rodriguez-Viejo, F. V. Mikulec, J. R. Heine, H. Mattoussi, R. Ober, K. F. Jensen and M. G. Bawendi, "(CdSe)ZnS Core-Shell Quantum Dots: Synthesis and Characterization of a Size Series of Highly Luminescent Nanocrystallites," Journal of Physical Chemistry B, Vol. 101, No. 46, 1997, pp. 9463-9475.

[8] T. Pons, I. L. Medintz, X. Wang, D. S. English, and H. Mattoussi, "Solution-Phase Single Quantum Dot Fluorescence Resonance Energy Transfer," Journal of American Chemical Society, Vol. 128, No. 47, 2006, pp. 15324 15331. doi:10.1021/ja0657253

[9] J. N. Miller, "Fluorescence Energy Transfer Methods in Bioanalysis," Analyst, Vol. 130, No. 3, 2005, pp. 265-270. doi:10.1039/b314346p

[10] S. Mayor and S. Bilgrami, "Fretting about FRET in Cell and Structural Biology," In: D. Zuk, Ed., Evaluating Techniques in Biochemical Research, Cell Press, Cambridge, 2007.

http://www.cellpress.com/misc/page?page=ETBR.

[11] U. Resch-Genger, M. Grabolle, S. Cavaliere-Jaricot, R. Nitschke and T. Nann, "Quantum Dots versus Organic Dyes as Fluorescent Labels," Nature Methods, Vol. 5, No. 9, 2008, pp. 763-775. doi:10.1038/nmeth.1248

[12] L. H. Qu and X. G. Peng, "Control of Photoluminescence Properties of CdSe Nanocrystals in Growth," Journal of American Chemical Society, Vol. 124, No. 9, 2002, pp. 2049-2055. doi:10.1021/ja017002j

[13] T. Forster, "10th Spiers Memorial Lecture. Transfer Mechanics of Electronic Excitation," Discussions of the Faraday Society, Vol. 27, 1959, pp. 7-17. doi:10.1039/df9592700007

[14] A. M. Dennis and G. Bao, "Quantum Dot-Fluorescent Protein Pairs as Novel Fluorescence Resonance Energy Transfer Probes," Nano Letters, Vol. 8, No. 5, 2008, pp. 1439-1445. doi:10.1021/n1080358+

[15] S. V. Dezhurov, I. Y. Volkova and M. S. Wakstein, "FRET-Based Biosensor for Oleic Acid in Nanomolar Range with Quantum Dots as an Energy Donor," Bioconjugate Chemistry, Vol. 22, No. 3, 2011, pp. 338-345.

[16] D. M. Willard, L. L. Carillo, J. Jung and A. V. Orden, "CdSe-ZnS Quantum Dots as Resonance Energy Transfer Donors in a Model Protein-Protein Binding Assay," Nano Letters, Vol. 1, No. 9, 2001, pp. 469-474. doi:10.1021/n1015565n

[17] G. Morello, M. Anni, P. D. Cozzoli, L. Manna, R. Cingolani and M. De Giorgi, "Picosecond Photoluminescence Decay Time in Colloidal Nanocrystals: The Role of Intrinsic and Surface States," Journal of Physical Chemistry C, Vol. 111, No. 28, 2007, pp. 10541-10545. doi:10.1021/jp072783h

[18] E. Mutlugün, S. Nizamoğlu and H. Volkan Demirb, "Highly Efficient Nonradiative Energy Transfer Using 
Charged CdSe/ZnS Nanocrystals for Light-Harvesting in Solution," Applied Physics Letters, Vol. 95, No. 3, 2009, p. 33106. doi: $10.1063 / 1.3182798$

[19] M. Zhou and I. Ghosh, "Current Trends in Peptide Science Quantum Dots and Peptides: A Bright Future Together," Biopolymers (PeptideScience), Vol. 88, No. 3, 2006, pp. 325-339. doi:10.1002/bip.20655

[20] T. Forster, "Zwischenmolekulare Energiewanderung und Fluoreszenz," Annal Physics, Vol. 437, No. 1-2, 1948, pp, $55-75$.

[21] R. M. Clegg, "Fluorescence Resonance Energy Transfer," In: X.-F. Wang and B. Herman, Eds., Fluorescence Imaging Spectroscopy and Microscopy, 1996, Wiley, New York, pp. 179-252.

[22] J. Lackowicz, "Principles of Fluorescence Spectroscopy," 3rd Edition, Springer Science and Business Media, LLC, New York.

[23] R. C. Somers, M. G. Bawendi and D. G. Nocera, "CdSe Nanocrystal Based Chem-/Bio- Sensors," Chemical Society Reviews, Vol. 36, No. 4, 2007, pp. 579-591. doi: $10.1039 / \mathrm{b} 517613 \mathrm{c}$

[24] A. L. Rogach, T. A. Klar, J. M. Lupton, A. Meijerink and J. Feldmann, "Energy Transfer with Semiconductor Nanocrystals," Journal of Materials Chemistry, Vol. 19, 2009, pp. 1208-1221. doi:10.1039/b812884g

[25] A. R. Clapp, I. L. Medintz and H. Mattoussi, "Forster Resonance Energy Transfer Investigations Using Quantum-Dot Fluorophores," ChemPhysChem, Vol. 7, No. 1, 2006, pp. 47-57. doi:10.1002/cphc.200500217

[26] C. S. Yun, A. Javier, T. Jennings, M. Fisher, S. Hira, S. Peterson, B. Hopkins, N. O. Reich and G. F. Strouse, "Nanometal Surface Energy Transfer in Optical Rulers, Breaking the FRET Barrier," Journal of American Chemical Society, Vol. 127, No. 9, 2005, pp. 3115-3119. doi:10.1021/ja043940i

[27] A. R. Clapp, I. L. Medintz, J. Matthew Mauro, B. R. Fisher, M. G. Bawendi and H. Mattoussi, "Fluorescence Resonance Energy Transfer Between Quantum Dot Donors and Dye-Labeled Protein Acceptors," Journal of American Chemical Society, Vol. 126, No. 1, 2004, pp. 301-310. doi:10.1021/ja037088b

[28] V. R. Hering, G. Gibson, R. I. Schumacher, A. FaljoniAlario and M. J. Politi, "Energy Transfer between CdSe/ ZnS Core/Shell Quantum Dots and Fluorescent Proteins," Bioconjugate Chemistry, Vol. 18, No. 6, 2007, pp. 17051708. doi:10.1021/bc700147j

[29] H. Lu, O. Schops, U. Woggon and C. M. Niemeyer, "Self-Assembled Donor Comprising Quantum Dots and Fluorescent Proteins for Long-Range Fluorescence Resonance Energy Transfer," Journal American Chemical Society, Vol. 130, No. 14, 2008, pp. 4815-4827. doi:10.1021/ja078243f

[30] H. Mattoussi, J. Matthew Mauro, E. R. Goldman, G. P. Anderson, V. C. Sundar, F. V. Mikulec and M. G. Bawendi, "Self-Assembly of CdSe-ZnS Quantum Dot Bioconjugates Using an Engineered Recombinant Protein," Journal American Chemical Society, Vol. 122, No. 49, 2000, pp. 12142-12150. doi:10.1021/ja002535y
[31] I.L. Medintz, T. Pons, K. Susumu, K. Boeneman, A.M. Dennis, D. Farrell, J. R. Deschamps, J. S. Melinger, G. Bao and H. Mattoussi, "Resonance Energy Transfer Between Luminescent Quantum Dots and Diverse Fluorescent Protein Acceptors," Journal of Physical Chemistry C, Vol. 113, No. 43, 2009, pp. 18552-18561. doi:10.1021/jp9060329

[32] J.-H. Wang, T.-C. Liu, Y.-C. Cao, X.-F. Hua, H.-Q. wang, H.-L. Zhang, X.-Q. Li and Y.-D. Zhao, "Fluorescence Resonance Energy Transfer between FITC and Water-Soluble CdSe/ZnS Quantum Dots," Colloids \& Surfaces A: Physicochem. Engineering Aspects, Vol. 302, No. 1, 2007, pp. 168-173. doi:10.1016/j.colsurfa.2007.02.018

[33] J. F. Callan, A. P. De Silva, R. C. Mulrooney and B. Mc Caugha, "Luminescent Sensing with Quantum Dots," Journal of Inclusion Phenomena and Macrocyclic Chemistry, Vol. 58, No. 3-4, 2007, pp. 257-262. doi:10.1007/s10847-006-9152-8

[34] A. M. Smith, H. Duan, A. M. Mohs and S. Nie, "Bioconjugated Quantum Dots for in Vivo Molecular and Cellular Imaging," Advanced Drug Delivery Reviews, Vol. 60, No. 11, 2008, pp. 1226-1240. doi:10.1016/j.addr.2008.03.015

[35] J. W. Borst and A. J. W. G. Visser, "Fluorescence Lifetime Imaging Micrsopey in Life Sciences," Measurement Science and Technology, Vol. 21, No. 10, 2010, p. 102002. doi:10.1088/0957-0233/21/10/102002

[36] R. S. Knox and H. V. Amerongen, "Refractive Index Dependence of the Forster Resonance Excitation Transfer Rate," Journal of Physical Chemistry B, Vol. 106, No. 20, 2002, pp. 5289-5293. doi:10.1021/jp013927+

[37] A. Iqbal, S. Arslan, B. Okumus, T. J. Wilson, G. Giraud, D. G. Norman, T. Ha and D. M. Lilley, "Orientation Dependence in Fluorescent Energy Transfer Between Cy3 and Cy5 Terminally Attached to Double-Stranded Nucleic Acids," Proceedings of the National Academy of Sciences, Vol. 105, No. 32, 2008, pp. 11176-11181.

[38] R. Kumaran and P. Ramamurthy, "PET Suppression Acridinedione Dyes by Urea Derivatives in Water and Methanol," Journal of Physical Chemistry B, Vol. 110, No. 47, 2006, pp. 23783-23789. doi:10.1021/jp0628378

[39] Y. Ren, Q. Xin, X.-T. Tao, L. Wang, X.-Q. Yu, J.-X. Yang and M.-H. Jiang, "Novel Multibranched Organic Compounds with Enhanced Two-Photon Absorption Benefiting from the Strong Electronic Coupling," Chemical Physics Letters, Vol. 414, No. 1-3, 2005, pp. 253-258. doi:10.1016/j.cplett.2005.08.041

[40] M. Grabolle, M. Spieles, V. Lesnyak, N. Gaponik, A. Eychmuller and U. Resch-Genger, "Determination of the Fluorescence Quantum Yield of Quantum Dots: Suitable Procedures and Achievable Uncertainties," Analytical Chemistry, Vol. 81, No. 15, 2009, pp. 6285-6294. doi:10.1021/ac $900308 \mathrm{v}$

[41] X. Wang, L. Qu, J. Zhang, X. Peng and M. Xiao, "Surface-Related Emission in Highly Luminescent CdSe Quantum Dots," Nano Letters, Vol. 3, No. 8, 2003, pp. 1103-1106. doi:10.1021/n10342491

[42] J.-Y. Zhang, X.-Y. Wang and M. Xiao, "Modification of Spontaneous Emission from $\mathrm{CdSe} / \mathrm{CdS}$ Quantum Dots in the Presence of a Semiconductor Interface," Optics Let- 
ters, Vol. 27, No. 14, 2002, pp. 1253-1255. doi:10.1364/OL.27.001253

[43] M. G. Bawendi, P. J. Carroll, W. L. Wilson and L. E. Brus, "Luminescence Properties of CdSe Quantum Crystallites: Resonance between Interior and Surface Localized States," Journal of Chemical Physics, Vol. 96, No. 946, 1992, pp. 946-954. doi:10.1063/1.462114

[44] A. L. Rogach (Ed), Semiconductor Nanocrystal Quantum Dots, Synthesis, Assembly and Applications, Springer Wein, New York, 2008. doi:10.1007/978-3-211-75237-1

[45] T. Pons, I. L. Medintz, M. Sykora and H. Mattoussi, "Spectrally Resolved Energy Transfer Using Quantum
Dot Donor: Ensemble and Single Molecule Photoluminescence Studies," Physical Review B, Vol. 73, No. 24, 2006, p. 245302. doi:10.1103/PhysRevB.73.245302

[46] S. Dayal and C. Burda, "Surface Effects on Quantum-Dot Based Energy Transfer," Journal of American Chemical Society, Vol. 129, No. 25, 2007, pp. 7977-7981. doi: $10.1021 / \mathrm{ja} 071457 \mathrm{c}$

[47] A. K. Parchur, A. I. Prasad, A. A. Ansari, S. B. Raia and R. S. Ningthoujam, "Luminescence Properties of $\mathrm{Tb}^{3+}$ Doped CaMoO4 Nanoparticles: Annealing Effect, Polar Medium Dispersible, Polymer Film and Core-Shell Formation," Dalton Transactions, Vol. 41, No. 36, 2012, pp. 11032-11045. doi:10.1039/c2dt31257c 